\title{
PAPERS
}

\section{Expression of Fas ligand by human gastric adenocarcinomas: a potential mechanism of immune escape in stomach cancer}

\author{
M W Bennett, J O’Connell, G C O’Sullivan, D Roche, C Brady, J Kelly, J K Collins, \\ F Shanahan
}

\begin{abstract}
Background-Despite being immunogenic, gastric cancers overcome antitumour immune responses by mechanisms that have yet to be fully elucidated. Fas ligand (FasL) is a molecule that induces Fas receptor mediated apoptosis of activated immunocytes, thereby mediating normal immune downregulatory roles including immune response termination, tolerance acquisition, and immune privilege. Colon cancer cell lines have previously been shown to express FasL and kill lymphoid cells by Fas mediated apoptosis in vitro. Many diverse tumours have since been found to express FasL suggesting that a "Fas counterattack" against antitumour immune effector cells may contribute to tumour immune escape.

Aim-To ascertain if human gastric tumours express FasL in vivo, as a potential mediator of immune escape in stomach
\end{abstract} cancer.

Specimens-Thirty paraffin wax embedded human gastric adenocarcinomas.

Department of Medicine, Cork

University Hospital,

National University of

Ireland, Cork, Ireland

$\mathrm{M} \mathrm{W}$ Bennett

J O'Connell

D Roche

C Brady

J Kelly

J K Collins

F Shanahan

Department of Surgery, Mercy Hospital, National

University of Ireland, Cork

G C O'Sullivan

Correspondence to: Professor F Shanahan, Department of Medicine, Clinical Sciences Building, University Hospital, Cork, Ireland.

Accepted for publication 19 August 1998 it may be cancer progression. TUNEL staining disclosed a high level of cell death among lymphocytes infiltrating FasL positive areas of tumour.

Conclusions-Human gastric adenocarcinomas express the immune downregulatory molecule, FasL. The results suggest that FasL is a prevalent mediator of immune privilege in stomach cancer. (Gut 1999;44:156-162)

Keywords: Fas ligand; gastric cancer; immune escape; apoptosis; tumour; mRNA

Despite expression of tumour rejection antigens such as MAGE $1-3^{1}$ and the presence of tumour specific cytotoxic $\mathrm{T}$ cells, ${ }^{2}$ the immune system fails to contain gastric carcinoma. Evidence suggests that a poor local immune response contributes to the potential for lymph nodal metastatic spread of gastric tumours. ${ }^{3}$ The mechanisms by which gastric cancers overcome antitumour immunological responses are poorly understood.

Fas ligand (FasL) triggers apoptotic cell death of sensitive lymphoid cells which express its cell surface receptor (Apo-1/CD95). ${ }^{4}$ FasL mediated apoptosis contributes to the regulation of the immune system through its roles in tolerance acquisition, ${ }^{5} \mathrm{~T}$ cell activation induced cell death, ${ }^{6}$ and immune response termination. ${ }^{7}$ FasL expressed in indigenous cells of the eye ${ }^{8}$ and the testis ${ }^{9}$ mediates immune privilege by inducing apoptosis in infiltrating proinflammatory immunocytes. FasL has been shown to confer immunological privilege in tissue transplantation experiments. In rodents, successful allograft survival was obtained of FasL expressing tissues ${ }^{910}$ and of non-immune privileged cells (pancreatic islets) co-transplanted with FasL expressing cells. ${ }^{11}{ }^{12}$ Allograft transplantation of murine tumour cells stably transfected with the FasL gene showed that FasL can cause local suppression of both humoral and cellular allograft specific immune responses. ${ }^{13}$

The possibility that FasL is expressed by non-lymphoid tumours as a mediator of immune evasion was initially raised by our finding that colon cancer cell lines express

Abbreviations used in this paper: FasL, Fas ligand; TUNEL, terminal deoxynucleotidyl transferase mediated dUTP nick end labelling; TdT, terminal deoxynucleotidyl transferase; PBS, phosphate buffered saline; SSC, $1 \times 0.15 \mathrm{M} \mathrm{NaCl} / 0.015 \mathrm{M}$ sodium citrate; TIL, tumour infiltrating lymphocyte. 
Table 1 Tumour, node, metastases (TNM) classification of gastric adenocarcinomas

\begin{tabular}{|c|c|c|c|c|}
\hline \multirow[b]{2}{*}{ Histological type } & \multirow[b]{2}{*}{ Number } & \multicolumn{3}{|c|}{ Stage } \\
\hline & & $T$ & $N$ & $M$ \\
\hline \multicolumn{5}{|l|}{ Adenocarcinoma } \\
\hline & 2 & $\mathrm{~T} 1$ & No & M0 \\
\hline & 7 & $\mathrm{~T} 2$ & N1 & M0 \\
\hline & 4 & T3 & No & M0 \\
\hline & 5 & T3 & N1 & M0 \\
\hline & 1 & T3 & N1 & M1 \\
\hline & 2 & $\mathrm{~T} 4$ & N1 & M1 \\
\hline & 1 & $\mathrm{~T} 4$ & N1 & M0 \\
\hline & 1 & $\mathrm{~T} 5$ & N3 & M0 \\
\hline \multirow[t]{2}{*}{ Signet-ring cell } & & & & \\
\hline & 5 & $\mathrm{~T} 2$ & N1 & M0 \\
\hline
\end{tabular}

Table 2 Grade of differentiation of gastric adenocarcinomas

\begin{tabular}{lr}
\hline Poor $^{\star}$ & 15 \\
Moderate to poor & 4 \\
Moderate & 11 \\
\hline
\end{tabular}

*All signet-ring cell carcinomas $(n=5)$ examined were poorly differentiated.

functional FasL. ${ }^{14}$ Colon cancer cells could induce Fas mediated apoptosis of Fas sensitive but not Fas resistant Jurkat T cells in vitro. A "Fas counterattack" model of tumour immune escape was proposed, in which a cancer cell, by expressing FasL, may remove Fas sensitive immune effector cells by apoptosis. Evidence for this mechanism of tumour immune privilege has since been provided for several cancers: melanoma, ${ }^{15}$ hepatocellular carcinoma, ${ }^{16}$ lung cancer, ${ }^{17}$ astrocytoma, ${ }^{18}$ and liver metastases of colon adenocarcinomas ${ }^{19}$ have been shown to express FasL. Recently, FasL expression by human oesophageal carcinoma was found to be associated with increased cell death of tumour infiltrating lymphocytes (TILs). ${ }^{20}$

To date only gastrointestinal cancers of oesophageal and colonic origin have been shown to express FasL. The aim of this study was to establish if gastric adenocarcinomas also express FasL. Using immunohistochemistry and in situ hybridisation to localise both FasL protein and mRNA, we set out to determine if FasL is expressed in vivo by neoplastic gastric epithelial cells.

\section{Materials and methods \\ SPECIMENS}

Thirty human gastric adenocarcinomas of disparate pathological stages (table 1) were collected after surgical resections performed at the Mercy Hospital, Cork, following a protocol approved by the University Teaching Hospitals ethics committee. None of the patients had received chemo-, radio- or immuno-therapy before resection. Tumours were moderately ( $\mathrm{n}$ $=11)$, poorly $(\mathrm{n}=15)$, or moderately to poorly $(n=4)$ differentiated (table 2$)$. Of the poorly differentiated tumours, five were Signet ring cell carcinomas.

IMMUNOHISTOCHEMICAL DETECTION OF FasL PROTEIN

Paraffin wax embedded surgically resected tumour sections were deparaffinised in xylene and rehydrated before analysis. The slides were washed twice for five minutes in a wash buffer containing $50 \mathrm{mM}$ Tris $/ \mathrm{HCl}, \mathrm{pH} 7.6,50 \mathrm{mM}$ $\mathrm{NaCl}$, and $0.001 \%$ saponin. Endogenous peroxidase was quenched with $3.0 \%$ hydrogen peroxide in methanol for five minutes. The slides were then washed as before except that the wash buffer for this and all subsequent steps included $1 \%$ normal goat serum. They were then blocked for one hour in wash buffer containing 5\% normal goat serum. This was followed by a further wash and incubation overnight at $4^{\circ} \mathrm{C}$ with affinity purified rabbit polyclonal anti-human FasL specific IgG (Santa Cruz Biotechnology, Santa Cruz, California, USA) at $0.1 \mu \mathrm{g} / \mathrm{ml}$ in wash buffer. Antibody binding was localised using a biotinylated secondary antibody, avidin conjugated horseradish peroxidase, and diaminobenzidine substrate, contained within the Vectastain ABC detection kit (Vector Laboratories, Burlingame, California, USA). The immunising peptide to which the antibody was raised (FasL: N-terminal amino acids 260-279; Santa Cruz Biotechnology) was included at $1 \mu \mathrm{g} / \mathrm{ml}$ during primary antibody incubation as a direct internal competitive control for antibody specificity. In a separate control for specificity of the Santa Cruz FasL specific polyclonal antibody, we previously found that specific antisense oligonucleotide mediated inhibition of FasL expression ${ }^{14}$ converted SW620 cells from positive to negative by immunohistochemistry with this antibody. The FasL specificity of the Santa Cruz antibody has also been previously verified in immunohistochemistry by others, staining FasL mRNA positive but not FasL mRNA negative cell lines, ${ }^{19}$ and staining in concordance with other FasL specific monoclona $1^{17}$ and polyclona $1^{19}$ antibodies. FasL detection in the gastric tumours was confirmed by immunohistochemistry with a FasL specific monoclonal antibody (Pharmingen, San Diego, California, USA; clone G247-4) as described above. The monoclonal antibody was used at a concentration of 5 $\mu \mathrm{g} / \mathrm{ml}$, and an isotype matched control antibody was also used. Slides were counterstained with haematoxylin.

\section{GENERATION OF A FasL SPECIFIC RNA PROBE} (RIBOPROBE)

A digoxigenin labelled RNA hybridisation probe (344 bp) was generated corresponding to codons 96-210 of the human FasL cDNA sequence. The riboprobe was synthesised by in vitro transcription using digoxygenin-11-UTP and T7 RNA polymerase (BoehringerMannheim GmbH, Mannheim, Germany). Template for the in vitro transcription reaction was generated by polymerase chain reaction (PCR) amplification of a fragment (codons 96-210) of FasL cDNA using a proofreading thermostable polymerase (UlTma DNA polymerase; Perkin-Elmer, Norwalk, Connecticut, USA) and an antisense primer to which a T7 promoter sequence was added.

RNA was isolated from FasL expressing cells by homogenisation in guanidine thiocyanate (Sigma Chemical Co, St Louis, Missouri, USA) followed by phenol extraction and etha- 
nol precipitation. cDNA was synthesised using the avian myeloblastosis virus reverse transcriptase (Promega, Madison, Wisconsin, USA) and random hexanucleotide primers (Boehringer-Mannheim).

PCR was performed on the CDNA using the following sense and antisense primers respectively: FasL: dGGATTGGGCCTGGGGATGTTTCA and d[pT7]-TTGTGGCTCAGG GGCAGGTTGTTG. PCR primers were designed using the DNASTAR Lasergene Primerselect program (DNASTAR Inc, Madison, Wisconsin, USA). Primer pairs were chosen to span introns in the FasL genomic sequence, thus ensuring mRNA specific amplification. Primers that showed no significant homology to any other genes in the EMBL DNA sequence database were selected.

Thermal cycling (40 cycles) was as follows: denaturation at $96^{\circ} \mathrm{C}$ for 15 seconds; annealing at $55^{\circ} \mathrm{C}$ for 30 seconds; extension at $72^{\circ} \mathrm{C}$ for three minutes. Primers were used at a final concentration of $0.1 \mu \mathrm{M}$ each, dNTPs at 50 $\mu \mathrm{M}$, and $\mathrm{MgCl}_{2}$ at $1.5 \mathrm{mM}$. A total of $1.0 \mathrm{U}$ of UITma DNA polymerase was used per $50 \mu \mathrm{l}$ reaction. PCR product specificity was confirmed by restriction mapping.

This PCR amplified FasL cDNA fragment was used as template to synthesise a riboprobe by in vitro transcription using digoxygenin-11UTP and T7 RNA polymerase (BoehringerMannheim) according to the manufacturer's instructions. The nucleotide sequence of the FasL probe showed no significant homology to any other sequence in the EMBL DNA sequence database. An unlabelled riboprobe was also synthesised for use in competitive control hybridisations.

LOCALISATION OF FasL mRNA EXPRESSION BY IN SITU HYBRIDISATION

In situ hybridisation was performed on paraffin wax embedded human gastric tumour sections ( $4 \mu \mathrm{m}$ thick), mounted on aminopropylethoxysilane treated slides. Prehybridisation treatments involved two washes of five minutes each in (a) phosphate buffered saline (PBS), (b) $\mathrm{PBS} / 0.1 \mathrm{M}$ glycine, (c) $\mathrm{PBS} / 0.3 \%$ Triton $\mathrm{X}-100$, and (d) PBS again. Sections were digested for 30 minutes at $37^{\circ} \mathrm{C}$ with proteinase $\mathrm{K}(10 \mu \mathrm{g} / \mathrm{ml}$ in $100 \mathrm{mM}$ Tris/HCl$/ 50 \mathrm{mM}$ EDTA, $\mathrm{pH}$ 8.0), fixed for five minutes at $4^{\circ} \mathrm{C}$ in $4 \%$ paraformaldehyde/PBS and then acetylated twice for five minutes in fresh $0.25 \%$ acetic anhydride/0.1 M triethanolamine, $\mathrm{pH}$ 8.0. Sections were incubated at $37^{\circ} \mathrm{C}$ for 10 minutes in a prehybridisation buffer consisting of $50 \%$ deionised formamide in $4 \times$ SSC (where $1 \times \mathrm{SSC}$ is $0.15 \mathrm{M} \mathrm{NaCl} / 0.015 \mathrm{M}$ sodium citrate). Hybridisation was performed at $42^{\circ} \mathrm{C}$ overnight in hybridisation buffer $(50 \%$ formamide, $10 \%$ dextran sulphate, $1 \times$ Denhardt's reagent, $4 \times \mathrm{SSC}, 10 \mathrm{mM}$ dithiothreitol, $500 \mu \mathrm{g} / \mathrm{ml}$ yeast tRNA, and $1 \mathrm{mg} / \mathrm{ml}$ heat denatured herring sperm DNA) containing $1 \mathrm{ng} / \mu \mathrm{l}$ digoxigenin labelled riboprobe. After hybridisation, tissues were washed with increasing stringency to $0.1 \times \mathrm{SSC}$ at $37^{\circ} \mathrm{C}$. Hybridised probe was detected immunologically using alkaline phosphatase conjugated sheep anti- digoxigenin antibody (Boehringer-Mannheim) and visualised with nitro blue tetrazolium/5bromo-4-chloro-3-indolyl phosphate (NBT/ BCIP) (purple/black precipitating product). Control slides involved direct internal competitive inhibition of hybridisation. ${ }^{16}{ }^{21}$ This was performed exactly as described above except that a tenfold excess of unlabelled FasL specific riboprobe was added to the digoxigenin labelled FasL specific riboprobe before hybridisation. This resulted in direct competitive displacement of positive hybridisation leading to a marked reduction in signal intensity, confirming the FasL specificity of hybridisation.

LEUCOCYTE COMMON ANTIGEN (CD45) STAINING To identify TILs, CD45 staining was performed on consecutive paraffin wax embedded sections. After deparaffinisation and rehydration, sections were pretreated by microwave irradiation in $0.01 \mathrm{M}$ citrate buffer for five minutes at $370 \mathrm{~W}$. The sections were rapidly cooled by immersion in $0.1 \mathrm{M}$ PBS. The slides were then incubated with a mouse anti-human CD45 monoclonal IgG (clone 2B11+PD7/26; Dako Corp, Carpinteria, California, USA) at a dilution of 1:70 for one hour. All incubations were carried out at room temperature. Next the slides were washed for five minutes in $0.1 \mathrm{M}$ Tris buffered saline which was used for all washes. After incubation with a secondary rabbit anti-mouse IgG (Dako Corp) at a dilution of 1:25 for 30 minutes, washing was repeated. Alkaline phosphatase conjugated anti-alkaline phosphatase (APAAP) complex (DO651; Dako Corp), at a dilution of 1:50, was incubated on the slides for 30 minutes and they were washed again. To enhance staining, the secondary antibody incubation and APAAP complex incubation were repeated once as above, but the duration of the incubation steps was reduced to 10 minutes each. Alkaline phosphatase substrate solution was then incubated on the slides for 10 minutes. The phosphatase substrate used was Fast Red (Sigma Chemical Co, St Louis, MO, USA). Slides were counterstained with haematoxylin and viewed by light microscopy.

CELL DEATH DETECTION IN SITU BY TERMINAL DEOXYNUCLEOTIDYL TRANSFERASE (TdT) MEDIATED dUTP NICK END LABELLING (TUNEL) Cell death was detected in situ in resected tissues by enzymic labelling of DNA strand breaks using a TUNEL assay (BoehringerMannheim) according to the manufacturer's instructions. Paraffin wax embedded surgically resected tumour sections were deparaffinised in xylene and rehydrated before analysis. After treatment with proteinase $\mathrm{K}(20 \mu \mathrm{g} / \mathrm{ml}$ in 10 $\mathrm{mM}$ Tris/ $\mathrm{HCl}, \mathrm{pH}$ 7.6) for 30 minutes, sections were washed in PBS and endogenous peroxidase activity was blocked in $0.3 \%$ hydrogen peroxide in methanol for 30 minutes. Next, the slides were washed. This and all subsequent washes were in PBS. The sections were treated with permeabilisation solution $(0.1 \%$ Triton $\mathrm{X}-100$ in $0.1 \%$ sodium citrate) for 15 minutes. After a wash, the labelling reaction was performed using a solution containing $\mathrm{TdT}$, its 
buffer and fluorescein conjugated dUTP. During this step, slides were coverslipped and incubated at $37^{\circ} \mathrm{C}$ for 60 minutes in a humidity chamber. TdT was omitted from negative control slides. To localise cells containing labelled DNA strand breaks, sections were washed and incubated with a sheep antifluorescein antibody $\mathrm{Fab}$ fragment conjugated with horseradish peroxidase at $37^{\circ} \mathrm{C}$ in a humidity chamber for 30 minutes. After a wash, colour was developed by incubating sections with a diaminobenzidine substrate solution for 15 minutes. After a final wash, sections were viewed by light microscopy. Only those cells with positive TUNEL staining and of apoptotic morphology were considered apoptotic.

\section{Results}

EXPRESSION OF FaSL PROTEIN BY GASTRIC ADENOCARCINOMAS

With the use of an affinity purified FasL specific rabbit polyclonal IgG (Santa Cruz Biotechnology) raised against a synthetic FasL peptide (N-terminal amino acids 260-279), FasL expression by tumour cells was immunohistochemically detected in all (30/30) surgically resected gastric adenocarcinomas (fig 1A). FasL specificity was confirmed using the FasL peptide immunogen (FasL amino acids 260-279) as an internal competitive control. Inclusion of the soluble peptide immunogen during immunohistochemistry resulted in direct competitive displacement of positive staining in consecutive control sections (fig 1B). The FasL specificity of the Santa Cruz polyclonal antibody used (C-20) had been previously verified by us ${ }^{14}$ and others. ${ }^{17}{ }^{19}$ FasL detection in the gastric tumours was confirmed using immunohistochemistry with a FasL specific monoclonal antibody (Pharmingen; FasL clone G247-4), which resulted in a pattern of staining identical with that detected with the Santa Cruz polyclonal antibody (not shown). An isotype-matched monoclonal antibody did not stain in control sections.

Positive staining of neoplastic tissue varied in intensity and extent, both within individual tumours and between tumours. Intensity of staining varied from weakly positive neoplastic areas to intensely staining tumour regions, where staining was stronger than that observed in local FasL positive TILs. FasL staining intensity was locally uniform within nests of tumour cells. FasL positive and negative staining neoplastic areas were frequently found to occur within the same tumour. However, all tumours examined were predominantly FasL positive $(>70 \%$ of tumour area).

LOCALISATION OF FasL mRNA TO NEOPLASTIC GASTRIC EPITHELIAL CELLS

A digoxigenin labelled FasL specific riboprobe was synthesised using the T7 RNA polymerase and a PCR generated cDNA template. The nucleotide sequence of the FasL riboprobe showed no significant homology to any other sequence within the EMBL DNA sequence database. Using in situ hybridisation with this probe, FasL mRNA expression was detected in tumour cells in resected gastric adenocarcinomas. Areas of positive hybridisation occurred within neoplastic cells throughout large areas of the tumours (fig 1C). The pattern of FasL mRNA detection corresponded closely to that of FasL immunostaining performed on consecutive tumour sections. Co-localisation of FasL mRNA and protein confirmed that the transformed gastric epithelial cells expressed FasL. Hybridisation was also detected in cells of lymphoid morphology - that is, TILs. Hybridisation specificity was confirmed by adding an excess of unlabelled FasL specific riboprobe to the digoxigenin labelled FasL specific riboprobe as an internal competitive control during hybridisation. This resulted in direct competitive displacement of positive hybridisation signals in consecutive control sections (fig 1D).

APOPTOSIS OF TILs

CD45 immunohistochemistry showed immunocyte infiltration in all 30 carcinomas (fig $2 \mathrm{~B}$ ). Tonsil sections included as positive controls stained strongly, and omission of the primary antibody abolished staining. Most of the CD45 positive cells were of lymphoid morphology. Recently, FasL expression by human oesophageal carcinoma was found to be associated with increased cell death of TILs. ${ }^{20}$ Apoptosis was detected by TUNEL among TILs adjacent to FasL positive areas of gastric carcinomas. These TUNEL positive TILs exhibited morphological features of apoptosis, including nuclear condensation and fragmentation (fig 2C). This was a consistent finding in all the tumours examined $(n=8)$.

\section{Discussion}

In this report, we show that stomach cancers express FasL, an inducer of immunocyte apoptosis. Expression of FasL potentially enables stomach tumours to counterattack and kill Fas sensitive antitumour immune effector cells (fig 3). We have previously shown that FasL expressed by colon adenocarcinoma cells in vitro is biologically active: SW620 cells induced Fas mediated apoptosis of co-cultured Jurkat $\mathrm{T}$ cells. As an established mediator of immune privilege and immunological tolerance in the eye ${ }^{8}$ and testis, ${ }^{9}$ our finding that gastric tumours express FasL suggests that FasL contributes to the immune evasion of stomach cancer.

Expression of FasL in vivo in human gastric cancers was prevalent; all 30 resected gastric adenocarcinomas were found to express FasL mRNA and protein. Co-localisation of FasL mRNA and protein confirmed that FasL was expressed by gastric epithelial tumour cells. Since activated lymphocytes are known to shed FasL, ${ }^{22}$ confirmation of tumour cell expression of FasL mRNA precludes the possibility that the detected FasL protein was derived from TILs. FasL staining was variable, in both intensity and extent, within tumours. Extensive expression $(>70 \%$ of the tumour area) occurred in all tumours irrespective of tumour stage or degree of differentiation (tables 1 and 2 respectively), suggesting that FasL may be 
expressed throughout gastric tumour progression. Whereas we found that FasL was undetectable by in situ hybridisation in control normal gastric epithelial sections $(\mathrm{n}=7$; not shown), FasL protein could be detected using
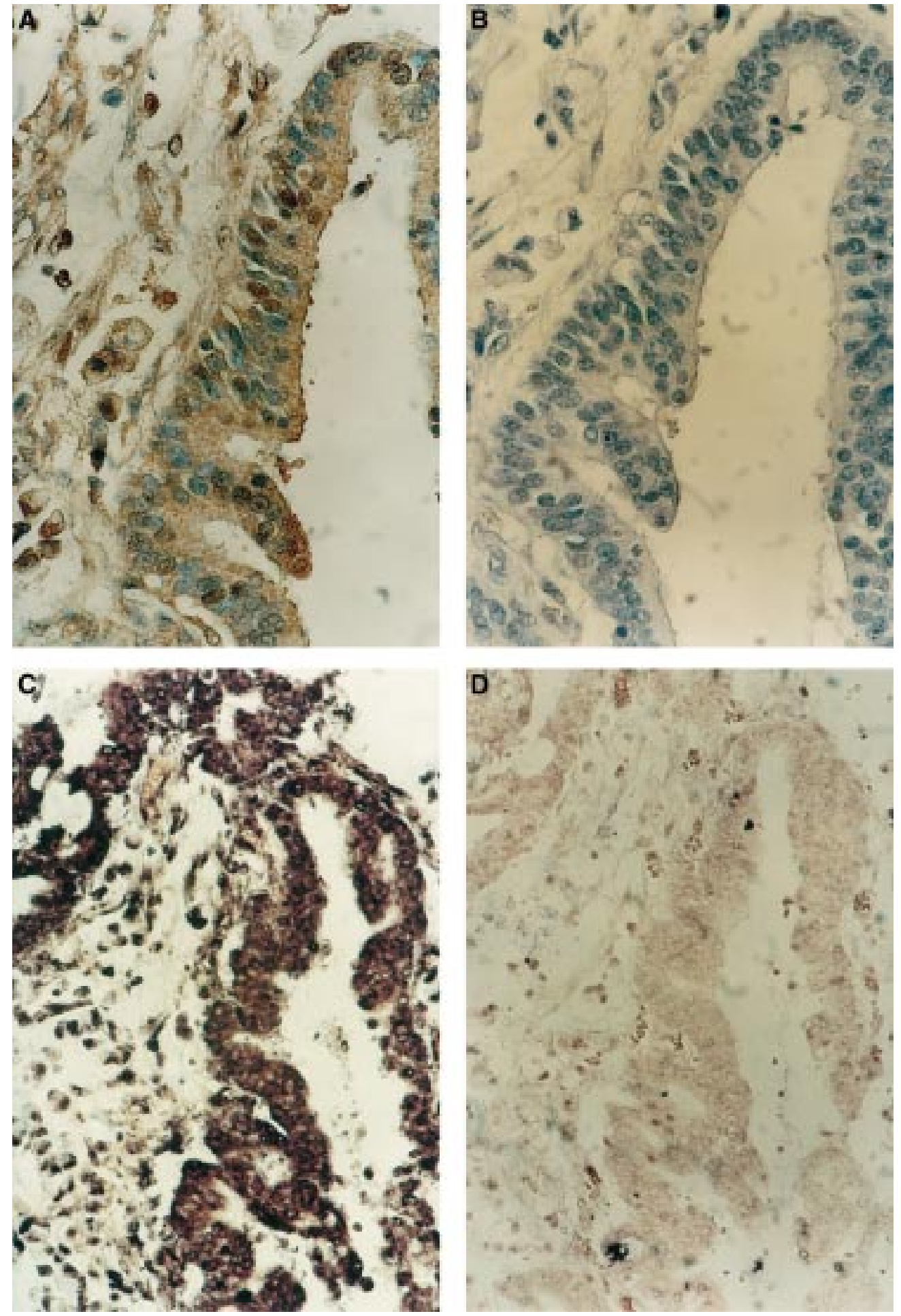

Figure 1 Expression of Fas ligand (FasL) in human gastric adenocarcinomas. Immunoperoxidase staining using a FasL specific rabbit polyclonal IgG was performed on paraffin wax embedded gastric carcinoma sections. Slides were counterstained with haematoxylin. (A) FasL positive immunohistochemical staining (brown) is shown in a representative gastric adenocarcinoma. (B) As a control for specificity of antibody detection, the FasL immunising peptide was included during primary antibody incubation. Competitive displacement of staining by the immunising peptide confirms FasL specificity. Tumour sections sequential to those shown in (A) and (B) were used to detect FasL mRNA by in situ hybridisation, using a digoxigenin labelled FasL specific riboprobe. (C) Positive purple hybridisation signal was obtained from the tumour area that stained immunohistochemically positive for FasL protein. (D) In a control hybridisation, a tenfold excess of unlabelled probe caused direct competitive displacement of the labelled probe, confirming the specificity of hybridisation. These results are representative of 30 adenocarcinomas of the stomach.

both FasL specific monoclonal and polyclonal antibodies (7/7). In contrast with the extensive detected in the gastric carcinon consistently restricted to only those epithelial

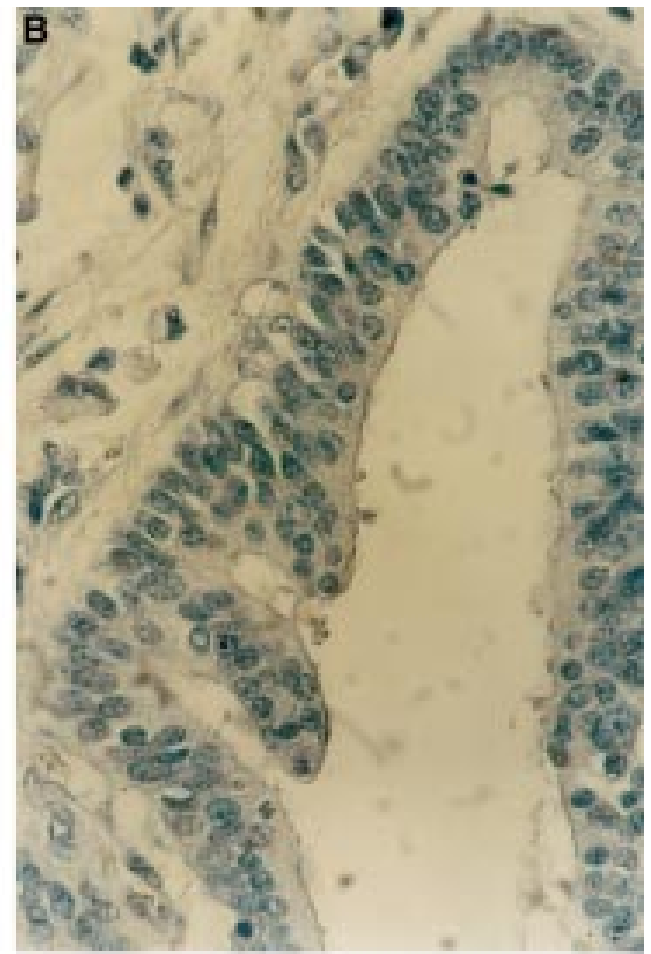



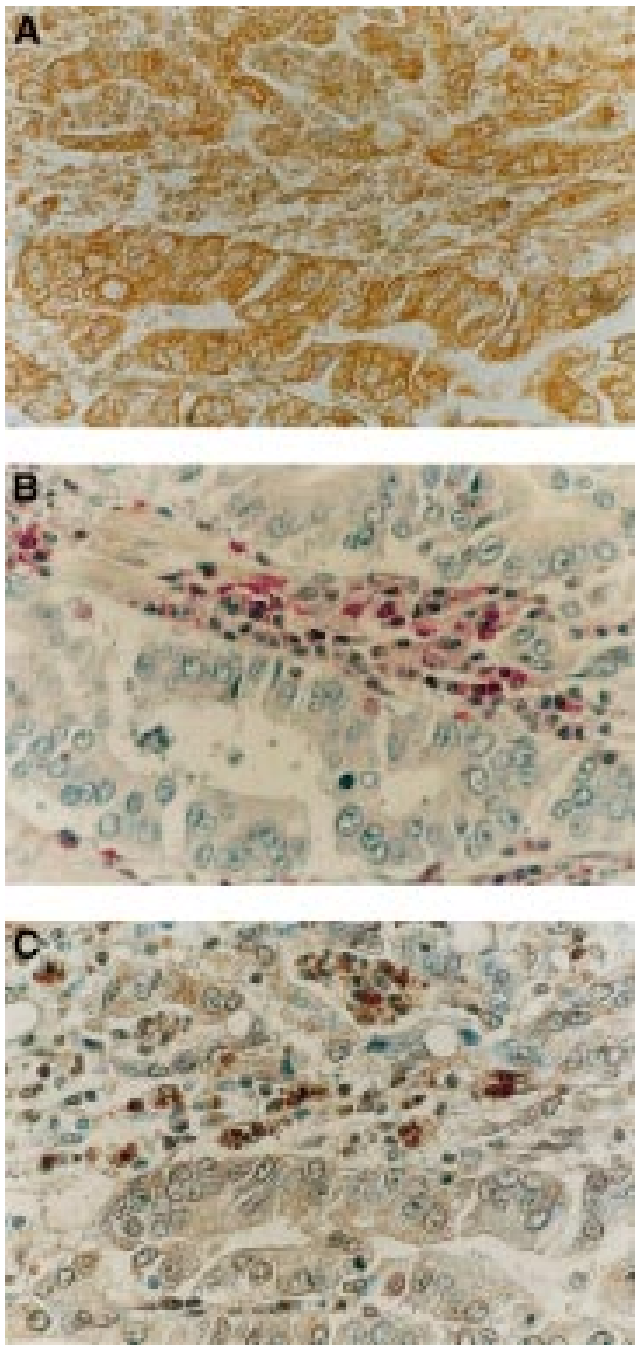

Figure 2 Apoptotic lymphocytes adjacent to FasL expressing carcinoma. (A) FasL expression (brown) detected in an area of gastric carcinoma by

immunoperoxidase staining. (B) CD45 leucocyte common antigen) immunophosphatase (alkaline phosphatase conjugated anti-alkaline phosphatase (APAAP)) staining (red) was performed on a sequential section. CD45 positive cells (red) of lymphoid morphology are present adjacent to areas of carcinoma. Slides were counterstained with haematoxylin. Isotype matched control sections were negative (not shown). (C) Cell death detection in situ by terminal deoxynucleotidyl transferase mediated dUTP nick end labelling (TUNEL). Tumour sections sequential to

those shown in $(A)$ and $(B)$ were used to detect cell death by enzymic labelling of DNA strand breaks using TUNEL. Only those cells with positive TUNEL staining (brown) and exhibiting apoptotic morphology were considered apoptotic. Control sections (not shown) where the labelling enzyme was omitted were negative.

cells at the luminal surface (7/7). Hence a deregulation and upregulation of FasL expression occurs during the transformation process.

A role for FasL in tumour immune escape is supported by the many downregulatory roles that this molecule normally plays in immunity. ${ }^{23}$ FasL is involved in mediating immune privilege in the eye, ${ }^{8}$ testis, ${ }^{9}$ and placenta, ${ }^{24}$ in contributing to immunological tolerance in the periphery, ${ }^{5}$ in downsizing immune responses, ${ }^{67}$ and in supporting experimental allograft survival in most cases. ${ }^{10-13}$ All of these roles involve FasL mediated apoptosis of lymphocytes. FasL has broad immunosuppressive effects: activated $\mathrm{T},{ }^{6} \mathrm{~B},{ }^{7}$ and $\mathrm{NK}^{25}$

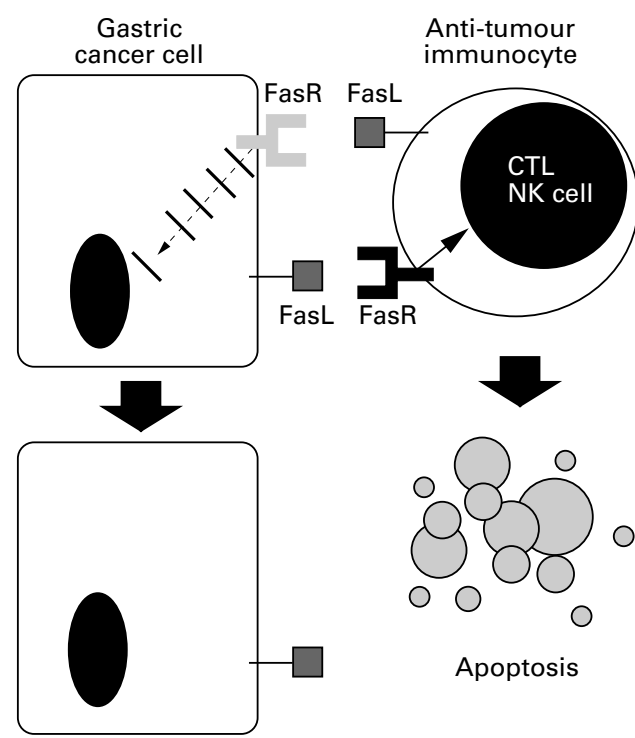

No apoptosis

Figure 3 The Fas counterattack. By expressing functional Fas ligand (FasL), gastric cancer cells can potentially counterattack and cause apoptosis of Fas sensitive tumour infiltrating immunocytes, which include antitumour $T$ (CTL) and NK cells. FasR, Fas receptor. As the result of various acquired defects in Fas signal transduction, most tumours are themselves relatively resistant to FasL mediated apoptosis. ${ }^{23}$

cells, neutrophils, ${ }^{26}$ and monocytes ${ }^{13}$ have all been shown to be sensitive to FasL mediated apoptosis. Surprisingly, in a few experiments, allografts of murine pancreatic islets ${ }^{27}$ or tumour cells ${ }^{29} 30$ genetically manipulated to express FasL were damaged as the result of proinflammatory neutrophil infiltration, for reasons that are as yet unclear. ${ }^{31}$ Conversely, adenovirus mediated overexpression of FasL in mouse ankle joints ameliorated collagen induced arthritis, providing powerful evidence for an anti-inflammatory role for FasL in vivo. ${ }^{32}$ Significant neutrophil infiltration was absent from all FasL expressing areas of the gastric tumours examined, suggesting that, in the context of spontaneous human gastrointestinal cancers, FasL expression mediates immune privilege rather than proinflammatory neutrophil recruitment.

Several cancers have been reported to express FasL: melanoma, ${ }^{15}$ hepatocellular carcinoma, ${ }^{16}$ lung cancer, ${ }^{17}$ astrocytoma, ${ }^{18}$ and liver metastases of colon adenocarcinomas. ${ }^{19}$ In all cases, tumour derived cell lines induced apoptosis in Fas sensitive, but not Fas resistant co-cultured lymphoid target cells in vitro. Tumours and cell lines themselves usually exhibit resistance to FasL mediated apoptosis because of various acquired defects in Fas signal transduction. ${ }^{23}$

Evidence that FasL inhibits tumour rejection in vivo comes from studies using a murine FasL expressing melanoma cell line. ${ }^{15}$ When injected into syngeneic host mice, this cell line quickly developed tumours. In syngeneic hosts expressing the defective mutant Fas receptor (lpr, or lymphoproliferation), tumour formation was retarded. The greater efficiency of tumour restriction by these syngeneic-lpr mice may have been due to the insensitivity of their 
lymphocytes to tumour expressed FasL. Although other immune escape mechanisms allowed the eventual establishment of tumours in FasL insensitive $l p r$ mutant mice, these experiments showed that FasL contributed to the immune privilege of the tumour, expediting tumour formation in wildtype mice. A recent study involving allograft transplantation of murine tumour cells stably transfected with the FasL gene showed that FasL caused profound local suppression of both humoral and cellular allograft specific immune responses. ${ }^{13}$

Immunogenic tumour cells are probably subjected to a barrage of cell mediated cytotoxic antitumour immune assaults. The fact that most tumour cells are efficiently killed by LAK cells in vitro ${ }^{33}$ suggests that cancer cells probably exhibit some degree of susceptibility to cell mediated cytotoxic mechanisms. Expression of a molecule to defuse antitumour immune challenge clearly offers a protective advantage to tumour growth and development. As an established mediator of immunological tolerance and privilege, FasL is such a molecule. Our findings conclusively show, at both the mRNA and protein levels, that human gastric tumours express FasL. Stomach cancer may therefore be added to the growing list of malignancies that appear to be immunologically privileged through FasL expression. The high prevalence of FasL expression in the tumours suggests that this molecule may be critical to tumour immune privilege. In conclusion, the Fas counterattack appears to prevail as a potentially critical mechanism of immune privilege in human stomach cancer.

We are grateful to Dr Gary Lee, Pathology Department, Mercy Hospital, Cork for access to surgically resected tissues and for the use of pathology laboratory facilities, Regina Limmer for tissue sectioning, Jacqui Kelly for assistance with antibody tissue sectioning, Jacqui Kelly for assistance with antibody staining, and Jim O'Callaghan for technical assistance. We also wish to thank the Health Research Board of Ireland, the Cancer Research Appeal at the Mercy Hospital, Cork, Ireland and the financial support.

1 Inoue $\mathrm{H}$, Mori $\mathrm{M}$, Honda $\mathrm{M}$, et al. The expression of tumor-rejection antigen "MAGE" genes in human gastric tumor-rejection antigen "MAGE" genes in hum

2 Hoshino T, Seki N, Kikuchi M, et al. HLA class-I-restricted and tumor-specific CTL in tumor-infiltrating lymphocytes and tumor-specific CTL in tumor-infiltrating lymphocytes

3 Maehara Y, Tomisaki S, Oda S, et al. Lymph node metastasis and relation to tumor growth potential and local immune response in advanced gastric cancer. Int $\mathcal{f}$ Cance 1997;74:224-8.

4 Nagata S, Golstein P. The Fas death factor. Science 1995;267:1449-56.

5 Mountz JD, Zhou T, Bluethmann H, et al. Apoptosis defects analyzed in TcR transgenic and fas transgenic lpr mice. Int Rev Immunol 1994;11:321-42.

6 Alderson MR, Tough TW, Davis-Smith T, et al. Fas ligand mediates activation-induced cell death in human $\mathrm{T}$ lymphocytes. F Exp Med 1995;181:71-7.

7 Daniel PT, Krammer PH. Activation induces sensitivity toward APO-1 (CD95)-mediated apoptosis in human B cells. F Immunol 1994;152:5624-32.
8 Griffith TS, Brunner T, Fletcher SM, et al. Fas ligandinduced apoptosis as a mechanism of immune privilege. Science 1995;270:1189-92

9 Bellgrau D, Gold D, Selawry H, et al. A role for CD95 ligand in preventing graft rejection. Nature 1995;377:630-2.

10 Stuart PM, Griffith TS, Usui N, et al. CD95 ligand (FasL)induced apoptosis is necessary for corneal allograft survival. f Clin Invest 1997;99:396-402.

11 Korbutt GS, Elliott JF, Rajotte RV. Cotransplantation of allogeneic islets with allogeneic testicular cell aggregates allows long-term graft survival without systemic immunosuppression. Diabetes 1997;46:317-22.

12 Lau HT, Yu M, Fontana A, et al. Prevention of islet allograft rejection with engineered myoblasts expressing FasL in mice. Science 1996;270:109-12.

13 Arai H, Chan SY, Bishop DK, et al. Inhibition of the alloantibody response by CD95 ligand. Nat Med 1997;3:843-8.

14 O'Connell J, O'Sullivan GC, Collins JK, et al. The Fas counterattack: Fas-mediated T cell killing by colon cancer cells expressing Fas ligand. F Exp Med 1996;184:1075-82.

15 Hahne M, Rimoldi D, Schroter M, et al. Melanoma cell expression of Fas(Apo-1/CD95) ligand: implications for tumor immune escape. Science 1996;274:1363-6.

16 Strand S, Hofmann WJ, Hug H, et al. Lymphocyte apoptosis induced by CD95 (Apo-1/Fas) ligand-expressing tumor cells: a mechanism of immune evasion? Nat Med 1996;2: 1361-6.

17 Niehans GA, Brunner T, Frizelle SP, et al. Human lung carcinomas express Fas ligand. Cancer Res 1997;57:1007-12.

18 Saas P, Walker PR, Hahne M, et al. Fas ligand expression by astrocytoma in vivo: maintaining immune privilege in the brain? f Clin Invest 1997;99:1173-8.

19 Shiraki K, Tsuji N, Shioda T, et al. Expression of Fas ligand in liver metastases of human colonic adenocarcinomas. Proc Natl Acad Sci USA 1997;94:6420-5.

20 Bennett MW, O'Connell J, O'Sullivan GC, et al. FasL expression in oesophageal carcinoma is associated with depletion of tumor infiltrating lymphocytes. F Immunol 1998;160:5669-75.

21 Galle PR, Hofmann WJ, Walczak $\mathrm{H}$, et al. Involvement of the CD95 (APO-1/Fas) receptor and ligand in liver damage. $\mathcal{F}$ Exp Med 1995;182:1223-30.

22 Tanaka M, Suda T, Takahashi T, et al. Expression of the functional soluble form of human fas ligand in activated lymphocytes. EMBO f 1995;14:1129-35.

23 O'Connell J, Bennett MW, O'Sullivan GC, et al. The Fas counterattack: a molecular mechanism of tumor immune privilege. Mol Med 1997;3:294-300.

24 Hunt JS, Vassmer D, Ferguson TA, et al. Fas ligand is positioned in mouse uterus and placenta to prevent trafficking of activated leukocytes between the mother and the conceptus. F Immunol 1997;158:4122-8.

25 Eischen CM, Schilling JD, Lynch DH, et al. Fc receptorinduced expression of Fas ligand on activated NK cells facilitates cell-mediated cytotoxicity and subsequent autocrine NK cell apoptosis. F Immunol 1996;156:2693-9.

26 Liles WC, Kiener PA, Ledbetter JA, et al. Differential expression of Fas (CD95) and Fas ligand on normal human phagocytes: implications for the regulation of apoptosis in neutrophils. F Exp Med 1996;184:429-40

27 Allison J, Georgiou HM, Strasser A, et al. Transgenic expression of CD95 ligand on islet beta cells induces a granulocytic infiltration but does not confer immune privilege upon islet allograft. Proc Natl Acad Sci USA 1997;94: 3943-7

28 Kang S-M, Schneider DB, Lin Z, et al. Fas ligand expression in islets of Langerhans does not confer immune privilege and instead targets them for rapid destruction. Nat Med 1997;3:738-43.

29 Seino K, Kayagaki N, Okumura K, et al. Antitumor effect of locally produced CD95 ligand. Nat Med 1997;3:165-70.

30 Arai H, Gordon D, Nabel EG, et al. Gene transfer of Fas ligand induces tumor regression in vivo. Proc Natl Acad Sci USA 1997;94:13862-7.

31 Lau HT, Stoeckert CJ. FasL: too much of a good thing? Nat Med 1997;3:727-8.

32 Zhang $\mathrm{H}$, Yang Y, Horton JL, et al. Amelioration of collagen-induced arthritis by CD95 (Apo-1/Fas)-ligand gene transfer. F Clin Invest 1997;100:1951-7.

33 Rayner AA, Grimm EA, Lotze MT. Lymphokine-activated killer (LAK) cells: analysis of factors relevant to the immunotherapy of human cancer. Cancer 1985;55:1327. 